\title{
BMJ Open Impaired fasting glucose is associated with increased severity of subclinical coronary artery disease compared to patients with diabetes and normal fasting glucose: evaluation by coronary computed tomographic angiography
}

\begin{abstract}
Swaminatha Gurudevan, ${ }^{1}$ Pankaj Garg, ${ }^{2}$ Shaista Malik, ${ }^{2}$ Ramni Khattar, ${ }^{2}$ Farhood Saremi, ${ }^{2}$ Harvey Hecht, ${ }^{3}$ Anthony DeMaria, ${ }^{4}$ Jagat Narula ${ }^{3}$
\end{abstract}

To cite: Gurudevan S, Garg P, Malik S, et al. Impaired fasting glucose is associated with increased severity of subclinical coronary artery disease compared to patients with diabetes and normal fasting glucose: evaluation by coronary computed tomographic angiography. BMJ Open 2016;6:e005148. doi:10.1136/bmjopen-2014005148

- Prepublication history for this paper is available online. To view these files please visit the journal online (http://dx.doi.org/10.1136/ bmjopen-2014-005148).

SG and $P G$ contributed equally to this article.

Received 3 March 2014 Revised 18 June 2014 Accepted 20 June 2014

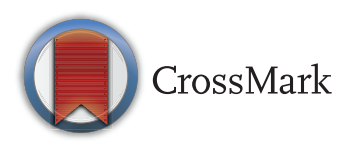

For numbered affiliations see end of article.

\section{Correspondence to} Dr Harvey Hecht; harvey.hecht@mountsinai.org

\section{ABSTRACT}

Objective: This study was designed to evaluate the severity of subclinical atherosclerosis in patients with asymptomatic impaired fasting glucose (IFG) compared to those with diabetes mellitus (DM) and normal fasting glucose (NFG), as measured by coronary computed tomographic angiography (CCTA).

Design: Subjects were divided into three groups: NFG (<100 mg/dL), IFG (100-125 mg/dL) and DM. Coronary artery calcium on non-contrast CT and plaque analysis on CCTA were performed.

Setting: University hospital, single centre.

Participants: 216 asymptomatic participants prospectively underwent CCTA for the evaluation of coronary artery disease (CAD).

Primary and secondary outcome measures: Atherosclerotic plaque burden in IFG compared to NFG patients.

Results: 2664 segments were analysed in 120 NFG, 44 IFG and 52 DM participants. The mean calcium scores were $178 \pm 395,259 \pm 510$ and $414 \pm 836$ for NFG, IFG and DM, respectively ( $p=0.037)$. The mean plaque burdens in the NFG, IFG and DM groups were 0.31 $\pm 0.45,0.50 \pm 0.69$ and $0.68 \pm 0.69$, respectively $(p=0.0007)$. A greater proportion of patients with DM $(19 / 52,36.5 \%)$ and IFG $(13 / 44,29.5 \%)$ had obstructive CAD compared to those with NFG (16/120, $13.3 \%)(p=0.0015)$. The number of segments with severe disease was significantly higher in the DM (60/ $637,9.4 \%)$ and IFG $(42 / 539,7.8 \%)$ groups compared to that in the NFG group $(34 / 1488,2.3 \%)(p=0.0001)$.

Conclusions: (1) IFG and DM have significantly higher, but comparable, calcium scores, plaque burden and obstructive CAD compared to NFG in asymptomatic individuals. (2) Pending corroboration by other reports, more intensive efforts may be devoted to the evaluation and treatment of patients with IFG.

While diabetes mellitus (DM) is considered a disease, there has been no similar classification for impaired fasting glucose (IFG),

\section{Strengths and limitations of this study}

- This is the first study to demonstrate comparable subclinical atherosclerosis in patients with DM and those with IFG.

- The study provides important implications for the large IFG population.

- The study has insufficient power, and not been designed, for clinical outcomes.

defined as a fasting blood glucose level of $100-126 \mathrm{mg} / \mathrm{dL}$, and also known as prediabetes. ${ }^{1}$ To examine its relationship with clinical coronary artery disease (CAD), a systematic literature review of 18 studies reported a significant but modest relative risk of $1.18-1.20 .^{2}$ In the symptomatic and asymptomatic population, there are numerous studies documenting the increased association of DM with subclinical atherosclerosis, ${ }^{3-10}$ but there are no data for pre-diabetes. Consequently, this study was designed to establish the role of IFG in plaque formation in asymptomatic patients compared to patients with DM and participants with normal fasting glucose (NFG), as measured by coronary computed tomographic angiography (CCTA).

\section{METHODS}

Patients

The study group consisted of 216 randomly selected, asymptomatic self-referred participants with no prior history of CAD, who voluntarily underwent MDCT between June 2005 and August 2007 as part of the investigational protocol for inclusion in this study. All participants had comprehensive 
evaluation of their cardiovascular risk factors through a questionnaire and a medical chart review along with laboratory assessment. Participants were separated into three groups based on their fasting plasma glucose. Individuals were classified as DM if they reported the condition or had fasting plasma glucose levels $>126 \mathrm{mg} / \mathrm{dL}$. IFG was defined as fasting plasma glucose levels between 100 and $126 \mathrm{mg} / \mathrm{dL}$; the remaining participants were designated as NFG.

\section{Coronary computed tomographic angiography}

All participants underwent coronary artery calcium (CAC) scoring and MDCT using a 64-slice scanner (Aquilion 64, Toshiba America Medical Systems, Tustin, California, USA). Oral $\beta$-blockers were administered by protocol to patients before the examination as needed to reduce heart rate. A $70-90 \mathrm{~mL}$ weight-based bolus of iohexol contrast agent (Omnipaque 350; Amersham Health, Ireland) was injected intravenously. When signal density in the descending aorta reached a predefined threshold of 180 Hounsfield units, the patient was instructed to sustain an inspiratory breath hold. The scan protocol used a gantry rotation time of $400 \mathrm{~ms}$, a slice collimation of $64 \times 0.5 \mathrm{~mm}$ applied at a table increment of $\sim 7.2 \mathrm{~mm} /$ rotation (depending on the patient's heart rate), a tube voltage of $120 \mathrm{kV}$ and a tube current of 300-500 mA (depending on the patient's weight). A scanning field of view of $320 \mathrm{~mm}$ was combined with a detector collimation of $64 \times 0.5 \mathrm{~mm}$ and a pixel matrix of $512 \times 512$. MDCT data were transferred to an image analysis workstation (Vitrea 2, Vital Images, Minnetonka, Minnesota, USA) and digitally rendered using an automated algorithm for cardiac structural analysis.

CAC scoring using the Agatston method was performed for each participant. For the CCTA data, a per-segment analysis of CAD was performed in each participant by a Level 3 Cardiac CT reader. Coronary artery segments were identified and classified in each participant based on the standard 15-segment AHA/WHO coronary nomenclature guidelines. ${ }^{11}$ For each visualised coronary artery segment, an ordinal scale was used to classify the degree of stenosis and atherosclerotic plaque: 0 for normal (no stenosis), 1 for mild plaque (stenosis $<30 \%$ ), 2 for moderate plaque (stenosis between $30 \%$ and $60 \%$ ) and 3 for obstructive plaque (stenosis $>60 \%$ ). The plaques were further characterised as calcified, non-calcified or mixed. Therefore, a total plaque burden score was determined in each participant by averaging the individual plaque scores over the total number of assessable segments. To calculate plaque burden score, each assessable segment was scored 0, 1, 2 or 3 . The total plaque burden score was the sum of all individual segment scores divided by the total number of assessable segments. The CCTA and CAC radiation doses were in the range of 5-10 and 1-2 $\mathrm{mSv}$, respectively.

\section{Statistics}

Statistical analysis was performed using PC-based software (Graphpad 5.0, San Diego, California, USA). Continuous variables were compared, including fasting plasma glucose, lipids, systolic blood pressure and plaque burden score in the DM, IFG and NFG groups, using the one-tailed analysis of variance (ANOVA). In addition to the three-group analysis, individual comparisons were also performed between the normal and IFG groups, normal and DM groups, and IFG and DM groups using the Mann-Whitney test for non-normally distributed variables and Student's t-test for normally distributed variables. To compare categorical variables in the per-segment analysis of coronary anatomy and the per-patient analysis of plaque burden, we used Pearson's $\chi^{2}$ test.

This study was approved by the Institutional Review Board.

\section{RESULTS}

\section{Demographics}

Of the 216 patients, 52 had DM, 44 had IFG and 120 had NFG.

The demographic characteristics in each group are shown in table 1 . There were no significant differences in age, smoking prevalence, statin use, family history of $\mathrm{CAD}$, and systolic and diastolic blood pressures among the three groups. When comparing DM with NFG, those with DM were older, had higher body mass index, lower low-density lipoprotein cholesterol (LDL) and higher prevalence of hypertension. When comparing the IFG group with the DM group, LDL was lower, triglycerides were higher and blood pressure was higher in those with DM than in those with IFG.

\section{Coronary artery calcium}

The mean calcium scores in the NFG, IFG and DM groups were $178 \pm 395,259 \pm 510$ and $414 \pm 836$, respectively. There was a significantly higher CAC $(p=0.01)$ in the DM group compared to the NFG group. Comparison between the IFG and DM groups and between the IFG and NFG groups revealed no significant differences ( $\mathrm{p}=0.2830$ and $\mathrm{p}=0.2860$, respectively). However, a three-group comparison using ANOVA demonstrated a significant trend of increasing CAC from NFG to IFG to DM $(p=0.04)$.

\section{Coronary computed tomographic angiography}

There was no statistically significant difference $(p=0.23)$ in plaque burden score in the DM $(0.68 \pm 0.69)$ and IFG $(0.50 \pm 0.69)$ groups (figure 1$)$. The IFG group's plaque burden was significantly higher than that seen in the NFG group $(0.31 \pm 0.45, p=0.04)$. Finally, comparison between the DM and NFG groups yielded a markedly higher plaque burden in the DM group $(p<0.0001)$.

\section{Per-segment analysis}

A total of 2664 coronary artery segments were analysed: 637 in the DM group, 539 in the IFG group and 1488 in the NFG group (table 2). Totally, 49 segments (19 DM $(3.0 \%), 13$ IFG (2.4\%), 17 NFG (1.1\%) and $1.8 \%$ 
Table 1 Demographics

\begin{tabular}{|c|c|c|c|c|c|c|c|}
\hline & DM & IFG & NFG & $\begin{array}{l}p \\
\text { (DM vs } \\
\text { IFG) }\end{array}$ & $\begin{array}{l}p \\
\text { (DM vs } \\
\text { NFG) }\end{array}$ & $\begin{array}{l}p \\
\text { (IFG vs } \\
\text { NFG) }\end{array}$ & $\begin{array}{l}\text { p } \\
\text { (3 Groups), } \\
\text { one-way } \\
\text { ANOVA or } \chi^{2}\end{array}$ \\
\hline Number & 52 & 44 & 120 & & & & \\
\hline Male & $30(57.7 \%)$ & $27(61.4 \%)$ & $79(65.8 \%)$ & & & & 0.5794 \\
\hline Female & $22(42.3 \%)$ & $17(38.6 \%)$ & $41(34.2 \%)$ & & & & \\
\hline Smokers & $13(25.0 \%)$ & $13(29.5 \%)$ & $28(23.3 \%)$ & & & & 0.7180 \\
\hline Age & $62.4 \pm 10.6$ & $59.4 \pm 12.7$ & $58.1 \pm 11.0$ & 0.2133 & 0.0183 & 0.5148 & 0.0735 \\
\hline $\mathrm{BMI}$ & $28.7 \pm 6.6$ & $27.4 \pm 5.5$ & $25.9 \pm 4.6$ & 0.3638 & 0.0057 & 0.1346 & 0.0182 \\
\hline Fasting glucose & $127.3 \pm 25.9$ & $107.3 \pm 5.5$ & $88.9 \pm 7.7$ & $<0.0001$ & $<0.0001$ & $<0.0001$ & $<0.0001$ \\
\hline \multicolumn{8}{|l|}{ Lipid profile } \\
\hline LDL & $90.5 \pm 34.2$ & $117.2 \pm 37.7$ & $114 \pm 37.1$ & 0.0023 & 0.0011 & 0.7034 & 0.0025 \\
\hline HDL & $45.4 \pm 13.7$ & $50.3 \pm 13.2$ & $52.1 \pm 17.9$ & 0.1229 & 0.0512 & 0.5496 & 0.1221 \\
\hline \multicolumn{8}{|l|}{ Blood pressure } \\
\hline Hx hypertension & 45 (86.5\%) & 27 (61.4\%) & $83(69.2 \%)$ & & & & 0.0113 \\
\hline Systolic BP & $146 \pm 19$ & $139 \pm 17$ & $138 \pm 19$ & 0.0830 & 0.0165 & 0.7301 & 0.0465 \\
\hline Diastolic BP & $81 \pm 11$ & $81 \pm 9$ & $79 \pm 10$ & 0.9763 & 0.2454 & 0.2626 & 0.3627 \\
\hline On statins & $18(34.6 \%)$ & $19(43.2 \%)$ & $46(38.3 \%)$ & & & & 0.6907 \\
\hline Family history of CAD & $19(36.5 \%)$ & $18(40.9 \%)$ & $35(29.2 \%)$ & & & & 0.3144 \\
\hline $\begin{array}{l}\mathrm{PAD} / \text { stroke/carotid } \\
\text { artery disease }\end{array}$ & $6(11.5 \%)$ & $1(2.3 \%)$ & $2(1.7 \%)$ & & & & 0.0093 \\
\hline
\end{tabular}

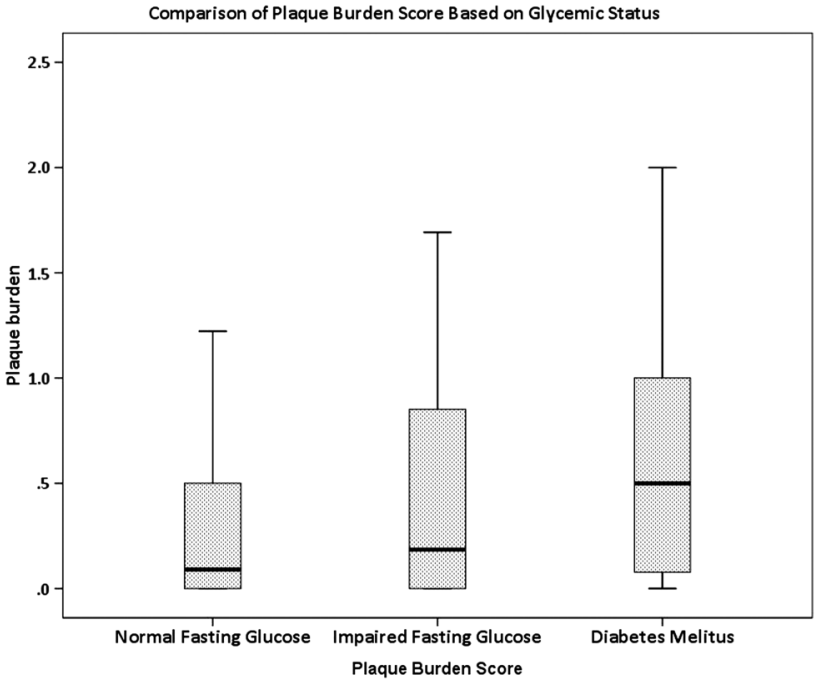

Figure 1 Plaque burden score in 216 patients separated according to their glycaemic status, with SEM shown in bars. Plaque burden was computed by scoring each assessable coronary segment as 0 for normal, 1 for mild plaque, 2 for moderate plaque and 3 for severe plaque and dividing by the number of assessable segments. ${ }^{*} p=0.04,{ }^{* *} p<0.0001$, $\ddagger \mathrm{p}=0.23$.

overall) could not be analysed due to motion artefacts or poor image quality, leaving a total of 2615 assessable segments $(98.2 \%)$. The NFG group had a significantly fewer diseased segments (any degree of stenosis) (22.6\%, $29.1 \%$ and $39.9 \%$, respectively, $\mathrm{p}<0.0001$ for both comparisons) compared to the IFG and DM groups. There were a significantly $(p<0.0001)$ greater number of segments with calcified plaques in participants with DM (26.7\%) compared to those with IFG (15.2\%) and NFG (14.6\%). The IFG and DM groups had a significantly greater proportion of non-calcified plaques than the NFG group, but there were no differences between the DM (6.3\%) and IFG (6.7\%) cohorts. Mixed plaques were noted in similar percentages in the three groups.

\section{Per-patient analysis}

The number of participants with obstructive CAD $(>60 \%$ stenosis $)$ was significantly $(\mathrm{p}=0.001)$ higher in the $\mathrm{DM}$ $(36.5 \%)$ and IFG $(29.5 \%)$ groups than that in the NFG group (13.3\%) (figure 2). When two-group comparisons were performed, there was no significant difference in the prevalence of obstructive CAD between the DM and IFG groups $(\mathrm{p}=0.52)$. There was a significantly higher proportion of individuals with obstructive CAD in the IFG versus NFG groups $(p=0.02)$ and the DM versus NFG groups ( $p=0.0009)$. In addition, the number of individuals with very high calcium scores $(>400)$ was significantly $(p=0.03)$ higher in the DM $(30.8 \%)$ and IFG $(18.2 \%)$ groups than that in the NFG group (13.3\%). In two-group comparisons, the DM group was significantly higher than the NFG group ( $p=0.01)$, but there was no significant difference between the DM compared to IFG groups $(p=0.24)$ and NFG compared to IFG groups $(\mathrm{p}=0.46)$.

\section{DISCUSSION}

This study is the first to provide evidence for the association of IFG, in addition to DM, with increased objective 
Table 2 Plaque severity and composition

\begin{tabular}{|c|c|c|c|c|c|c|c|}
\hline & DM & IFG & NFG & $\begin{array}{l}\text { p } \\
\text { (DM vs IFG) }\end{array}$ & $\begin{array}{l}p \\
\text { (IFG vs NFG) }\end{array}$ & $\begin{array}{l}p \\
\text { (DM vs NFG) }\end{array}$ & $\begin{array}{l}p \\
\text { (3 groups) }\end{array}$ \\
\hline \multicolumn{8}{|l|}{ Plaque severity } \\
\hline $\begin{array}{l}\text { Total segments } \\
\text { analysed }\end{array}$ & 637 & 539 & 1488 & & & & \\
\hline Normal segments & $383(60.1 \%)$ & $382(70.9 \%)$ & $1152(77.4 \%)$ & & & & $<0.001$ \\
\hline $\begin{array}{l}\text { Mild plaque } \\
\text { segments }\end{array}$ & $135(21.1 \%)$ & $81(15.0 \%)$ & $223(15.0 \%)$ & & & & \\
\hline $\begin{array}{l}\text { Moderate plaque } \\
\text { segments }\end{array}$ & 59 (9.3\%) & 34 (6.3\%) & 79 (5.3\%) & & & & \\
\hline $\begin{array}{l}\text { Severe plaque } \\
\text { segments }\end{array}$ & $60(9.4 \%)$ & $42(7.8 \%)$ & $34(2.3 \%)$ & & & & \\
\hline $\begin{array}{l}\text { Total diseased } \\
\text { segments } \\
\text { Plaque composition }\end{array}$ & 254 (39.9\%) & 157 (29.1\%) & $336(22.6 \%)$ & 0.0001 & 0.0029 & $<0.0001$ & $<0.0001$ \\
\hline Total plaques & 254 & 157 & 336 & & & & \\
\hline Calcified plaques & 170 (26.7\%) & $82(15.2 \%)$ & 218 (14.6\%) & 0.0035 & 0.0099 & $<0.0001$ & 0.0068 \\
\hline Mixed plaques & $44(6.9 \%)$ & $39(7.2 \%)$ & $73(4.9 \%)$ & 0.0766 & 0.4890 & 0.4060 & 0.1668 \\
\hline $\begin{array}{l}\text { Non-calcified } \\
\text { plaques }\end{array}$ & $40(6.3 \%)$ & $36(6.7 \%)$ & $45(3.0 \%)$ & 0.0877 & 0.00092 & $<0.0001$ & 0.0270 \\
\hline
\end{tabular}

measures of CAD compared to NFG, including significantly higher, but comparable, calcium scores, plaque burden and obstructive CAD in patients with asymptomatic IFG and DM compared to individuals with NFG. Although DM is generally regarded as a CAD equivalent, ${ }^{12}$ more recent data indicate that this may not be the case. A recent meta-analysis of 45108 patients reported that patients with DM and no myocardial infarction had $43 \%$ fewer events than patients with a prior myocardial infarction and no DM. ${ }^{13}$ In addition, it has become clear through CAC studies that patients with DM have the same spectrum of CAC scores as those with no DM, with, however, more risk at every CAC level, ${ }^{3-10}$ except for equivalent very low risk in those with $0 \mathrm{CAC}^{4}$
Whether these findings apply to prediabetes patients is unknown. Microvascular disease is not a sequel of IFG; several years of DM is necessary. ${ }^{1}$ IFG does impose an $18-20 \%$ increase in macrovascular cardiovascular risk, ${ }^{2}$ presumably mediated by insulin resistance and frequent association with the metabolic syndrome. The conversion rate to DM is $10 \%$ annually, thereby adding further to the rapid worldwide increase in DM prevalence, from 171 million in 2000 to a projected 366 million in $2030 .{ }^{14}$ The magnitude of the issue is apparent in the USA from the IFG prevalence of $25.7 \%$, with an estimated affected population of 57 million adults. ${ }^{1}$ Other countries are similarly afflicted, with $37.6 \%$ prevalence in Denmark and $37.6 \%$ in India. ${ }^{1}$ Therefore, in the setting of a
Figure 2 Percentage of patients studied with CACS $>400$ and obstructive CAD (at least one coronary artery segment with $>60 \%$ stenosis) separated based on their glycaemic status. ${ }^{*} \mathrm{p}=0.46$ and 0.02 for CACS $>400$ and obstructive CAD, respectively; ${ }^{* *} p=0.01$ and 0.0009 for CACS $>400$ and obstructive CAD, respectively; $\ddagger \mathrm{p}=0.24$ and 0.52 and for CACS $>400$ and obstructive CAD, respectively. CACS, coronary artery calcium score; CAD, coronary artery disease.
Prevalence of CAC $>\mathbf{4 0 0}$ and Obstructive CAD based on Glycemic Status

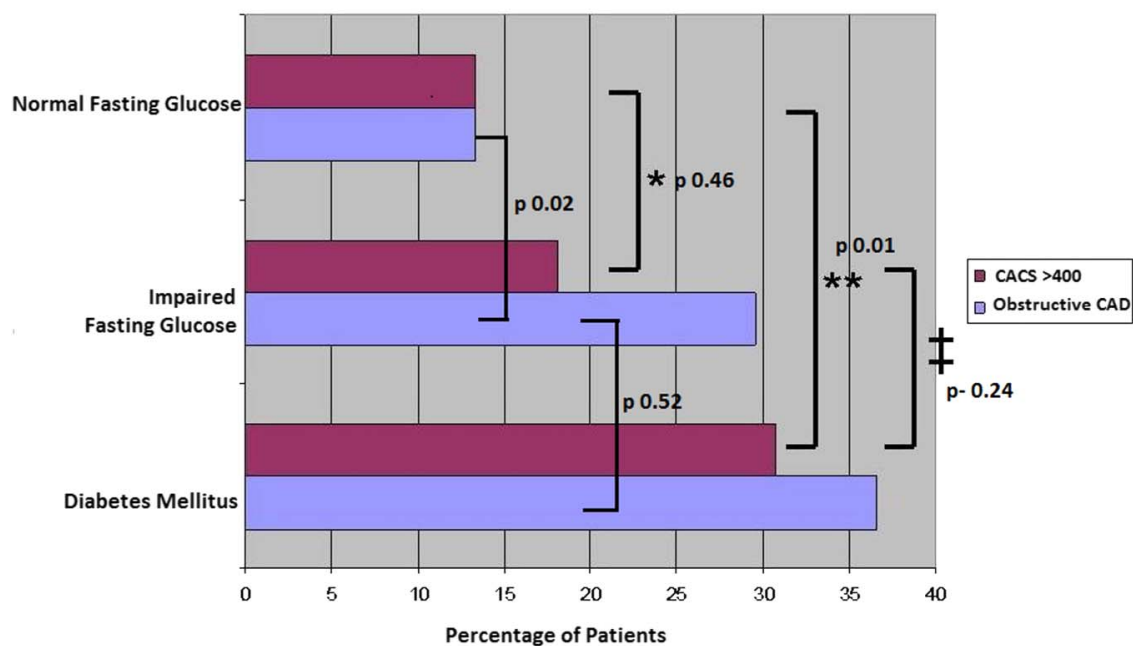


paucity of data and widespread prevalence, establishing the CAD risk in this large cohort is of major public health importance.

There are two prior studies correlating the degree of glycaemic derangement in diabetic participants with CAD burden by conventional angiography. Seibaek et al compared the results of glucose tolerance tests in a population of 99 patients with documented CAD to a control group of 112 patients without CAD. ${ }^{15}$ They found that a higher percentage of patients with CAD had abnormal glucose tolerance but were unable to demonstrate a specific correlation between the degree of hyperglycaemia and the amount of CAD. More recently, Quadros et al studied 145 individuals undergoing coronary angiography and divided them into four groups based on their fasting plasma glucose; they were able to demonstrate a good correlation between hyperglycaemia and CAD burden. ${ }^{16}$ Conventional angiography, however, is limited by its inability to identify the non-obstructive plaque burden. CCTA, on the other hand, is ideally suited to identify and semiquantify all plaque.

DM has been evaluated by CAC in multiple studies, and the increased prevalence of CAC in DM is well documented, as is the worse prognosis in those with CAC. ${ }^{3-10}$ In CCTA evaluations, Pundziute et a ${ }^{\ominus}$ noted more diseased segments, calcified and non-calcified plaques in 215 patients with symptomatic DM compared to individuals with no DM, and Scholte $e t a l^{10}$ in 70 patients with asymptomatic DM reported plaque in $80 \%$, $41 \%$ of which were non-calcified. Neither paper evaluated patients with IFG. Until the present study, there were no CAC or CCTA evaluations of patients with IFG. The findings are consistent with the near equivalency of subclinical atherosclerosis in the DM and IFG groups. With the exception of higher percentages of diseased segments, and calcified plaques in the DM group, all other measures of atherosclerosis, including calcium score, CAC $>400$, plaque burden, proportion of noncalcified and mixed plaques, and incidence of obstructive disease, were no different in the DM and IFG cohorts. In contrast, most of the parameters of atherosclerosis severity were worse in the IFG group compared to the NFG group; only the frequencies of mild, moderate and mixed plaques were similar. This study does not address the mechanism of the advanced atherosclerosis in the IFG group for which further research is needed.

\section{Weaknesses}

There were several limitations to this study. First, the prevalence of hypertension was slightly higher in the DM group, which may have made a small contribution to the greater burden of CAD in this group. Second, in this asymptomatic population, the presence of CAD on CCTA was used as a surrogate end point for clinical $\mathrm{CAD}$. However, this has been the rule in asymptomatic populations, in whom event rates are low and invasive angiography not warranted. Finally, this study was neither powered nor designed to examine clinical outcomes.

\section{Clinical significance}

While most physicians are familiar with the risks of DM and the metabolic syndrome, IFG remains under the radar. The near equivalency of subclinical atherosclerosis in DM and IFG should heighten awareness of the risks of IFG. Pending corroboration by other reports, more intensive efforts may be devoted to the evaluation by CAC scanning and treatment of patients with pre-diabetes.

Author affiliations

${ }^{1}$ Department of Cardiology, University of California at Los Angeles, Los Angeles, California, USA

${ }^{2}$ Department of Cardiology, University of California at Irvine, Irvine, California, USA

${ }^{3}$ Department of Cardiology, Icahn School of Medicine at Mount Sinai, New York, New York, USA

${ }^{4}$ Department of Cardiology, University of California at San Diego, San Diego, California, USA

Contributors SG recruited patients and performed all studies with PG. PG collected all data, database and first draft. SM involved in database analysis and paper writing with PG. RK made calls for follow-ups. FS performed all studies in association with $\mathrm{SG}$. $\mathrm{HH}$ and $\mathrm{AD}$ participated in discussion of results and paper editing. JN is the principal investigator, created the study plan, and participated in review of analysis and paper editing.

Funding This research received no specific grant from any funding agency in the public, commercial or not-for-profit sectors.

Competing interests None declared.

Ethics approval University of California Irvine IRB.

Provenance and peer review Not commissioned; externally peer reviewed.

Data sharing statement No additional data are available.

Open Access This is an Open Access article distributed in accordance with the Creative Commons Attribution Non Commercial (CC BY-NC 4.0) license, which permits others to distribute, remix, adapt, build upon this work noncommercially, and license their derivative works on different terms, provided the original work is properly cited and the use is non-commercial. See: http:// creativecommons.org/licenses/by-nc/4.0/

\section{REFERENCES}

1. Grundy SM. Pre-diabetes, metabolic syndrome and cardiovascular risk. J Am Coll Cardiol 2012;59:635-43.

2. Ford ES, Zhao G, Li C. Pre-diabetes and the risk for cardiovascular disease: a systematic review of the evidence. J Am Coll Cardiol 2010;55:1310-17.

3. Wong ND, Sciammarella MG, Polk D, et al. The metabolic syndrome, diabetes, and subclinical atherosclerosis assessed by coronary calcium. J Am Coll Cardiol 2003;41:1547-53.

4. Raggi $P$, Shaw LJ, Berman DS, et al. Prognostic value of coronary artery calcium screening in subjects with and without diabetes. J Am Coll Cardiol 2004;43:1663-9.

5. Anand DV, Lim E, Hopkins D, et al. Risk stratification in uncomplicated type 2 diabetes: prospective evaluation of the combined use of coronary artery calcium imaging and selective myocardial perfusion scintigraphy. Eur Heart J 2006;27:713-21.

6. Becker A, Leber A, Becker B. Predictive value of coronary calcifications for future cardiac events in asymptomatic patients with diabetes mellitus: prospective study in 716 patients over 8 years. BMC Cardiovasc Disord 2008;27:1-8.

7. Elkeles R, Godsland IF, Feher MD, et al. Coronary calcium measurement improves prediction of cardiovascular events in asymptomatic patients with type 2 diabetes: the PREDICT study. Eur Heart J 2008;29:2244-51.

8. Malik S, Budoff MJ, Katz R. Impact of subclinical atherosclerosis on cardiovascular disease events in individuals with metabolic syndrome and diabetes: the multi-ethnic study of atherosclerosis. Diabetes Care 2011;34:2285-90. 
9. Pundziute G, Schuijf JD, Jukema JW. Noninvasive assessment of plaque characteristics with multislice computed tomography coronary angiography in symptomatic diabetic patients. Diabetes Care 2007:30:1113-19.

10. Scholte AJ, Schuijf JD, Kharagjitsingh AV, et al. Prevalence of coronary artery disease and plaque morphology assessed by multi-slice computed tomography coronary angiography and calcium scoring in asymptomatic patients with type 2 diabetes. Heart 2008;94:290-5.

11. Austen WG, Edwards JE, Frye RL. A reporting system on patients evaluated for coronary artery disease. Report of the Ad Hoc Committee for Grading of Coronary Artery Disease, Council on Cardiovascular Surgery, American Heart Association. Circulation 1975;51(4 Suppl):5-40.

12. Haffner SM, Lehto $\mathrm{S}$, Ronnemaa $\mathrm{T}$, et al. Mortality from coronary heart disease in subjects with type 2 diabetes and in nondiabetic subjects with and without prior myocardial infarction. N Engl J Med 1998;339:229-34.

13. Bulagahapatiya $U$, Siyambalapitiya $J$, Sithole $\mathrm{J}$, et al. Is diabetes a coronary risk equivalent? Systematic review and meta-analysis. Diabetes Med 2009;26:143-8.

14. World Health Organization country and regional data on diabetes. 2014. http://www.who.int/diabetes/facts/world_figures/en/

15. Seibaek M, Sloth C, Vallebo L, et al. Glucose tolerance status and severity of coronary artery disease in men referred to coronary arteriography. Am Heart J 1997;133:622-9.

16. Quadros AS, Sarmento-Leite R, Bertoluci M, et al. Angiographic coronary artery disease is associated with progressively higher levels of fasting plasma glucose. Diabetes Res Clin Pract 2007;75:207-11. 